\title{
Prognostic and predictive value of TOPK stratified by KRAS and BRAF gene alterations in sporadic, hereditary and metastatic colorectal cancer patients
}

\author{
I Zlobec ${ }^{*, I}$, F Molinari ${ }^{2}$, M Kovac ${ }^{3}$, MP Bihl', HJ Altermatt ${ }^{4}$, J Diebold ${ }^{5}$, H Frick ${ }^{6}$, M Germer $^{7}$, M Horcic $^{8}$, \\ M Montani ${ }^{9}$, G Singer ${ }^{10}$, H Yurtsever ${ }^{11}$, A Zettl ${ }^{12}$, L Terracciano', L Mazzucchelli ${ }^{2}$, P Saletti ${ }^{13}, M^{1}$ Frattini $^{2}$, \\ K Heinimann ${ }^{3}$ and A Lugli'
}

'Institute for Pathology, University Hospital of Basel, Basel, Switzerland; ${ }^{2}$ Institute of Pathology, Locarno, Switzerland; ${ }^{3}$ Research Group Human Genetics, Department of Biomedicine, University of Basel, Basel, Switzerland; ${ }^{4}$ Pathologie Länggasse, Bern, Switzerland; ${ }^{5}$ Institute for Pathology, Cantonal Hospital of Lucerne, Lucerne, Switzerland; ' Institute for Pathology, Cantonal Hospital of Chur, Chur, Switzerland; ' Institute for Pathology, Cantonal Hospital of Winterthur, Winterthur, Switzerland; ${ }^{8}$ Institute for Histological and Cytological Diagnostics, Aarau, Switzerland; ${ }^{9}$ Department of Pathology, University Hospital Zurich, Zurich, Switzerland; ${ }^{10}$ Institute for Pathology, Cantonal Hospital of Baden, Baden, Switzerland; "Institute for Pathology, Cantonal Hospital of Aarau, Aarau, Switzerland; ${ }^{12}$ Institute of Clinical Pathology, Basel, Switzerland; ${ }^{13}$ Oncology Institute of Southern Switzerland, Ospedale San Giovanni, Bellinzona, Switzerland

\begin{abstract}
BACKGROUND: Our aim was to investigate the prognostic and predictive value of the oncogenic MAPKK-like protein T-cell-originated protein kinase (TOPK) stratified by KRAS and BRAF mutations in patients with sporadic, hereditary and metastatic colorectal cancer $(\mathrm{CRC})$ treated with anti-EGFR therapy.

METHODS: Immunohistochemistry (IHC) for TOPK was performed on four study groups. Group I included two subgroups of 543 and 50 I sporadic CRC patients used to test the reliability of TOPK expression by IHC. In Group 2, representing an additional 222 sporadic CRCs, the prognostic effect of TOPK stratified by KRAS and BRAF was assessed. The prognostic effect of TOPK was further analysed in Group 3, representing 7I hereditary Lynch syndrome-associated CRC patients. In Group 4, the predictive and prognostic value of TOPK was analysed on 45 metastatic patients treated with cetuximab or panitumumab stratified by KRAS and BRAF gene status. RESULTS: In both sporadic CRC subgroups (Group I), associations of diffuse TOPK expression with clinicopathological features were reproducible. Molecular analysis of sporadic CRCs in Group 2 showed that diffuse TOPK expression was associated with KRAS and BRAF mutations $(p<0.00 \mathrm{I})$ and with poor outcome in patients with either mutation in univariate and multivariate analysis $(P=0.0 \mathrm{I} 7)$. In hereditary patients (Group 3), diffuse TOPK was linked to advanced PT stage. In metastatic patients treated with anti-EGFR therapy (Group 4), diffuse TOPK expression was linked to dismal outcome despite objective response to treatment $(P=0.0 \mathrm{I})$.

CONCLUSION: TOPK expression is an unfavourable prognostic indicator in sporadic patients with KRAS or BRAF mutations and also in patients with metastatic disease experiencing a response to anti-EGFR therapies. The inhibition of TOPK, which could benefit $30-40 \%$ of CRC patients, may represent a new avenue of investigation for targeted therapy.

British Journal of Cancer (2010) 1 02, I5I-161. doi:10.1038/sj.bjc.6605452 www.bjcancer.com

Published online 24 November 2009

(c) 2010 Cancer Research UK
\end{abstract}

Keywords: colorectal cancer; TOPK; KRAS; BRAF; anti-EGFR therapy

The pathogenesis, progression and oncogenic behaviour of colorectal cancer (CRC) are to a large extent regulated by the ERK/ MAPK signalling cascade, which activates transcription factors critical for angiogenesis, proliferation, apoptosis, differentiation and metastasis (Fang and Richardson, 2005). In CRC, $30-40 \%$ of cases have mutations in the KRAS proto-oncogene (Bos et al, 1987). Often linked to tumours arising from the chromosomal instability pathway, representing $80-85 \%$ of CRC cases, KRAS mutations have been associated with increased activity of ERK signalling, thereby promoting transcription of Elk-1 and $c-M y c$

*Correspondence: Dr I Zlobec, Institute of Pathology, University of Basel, Schönbeinstrasse 40, Basel, 403I, Switzerland; E-mail: izlobec@uhbs.ch Revised 14 October 2009; accepted 22 October 2009; published online 24 November 2009
(Bos et al, 1987; Jass, 2007). Although evidence with regard to the effect of KRAS gene status on prognosis is heavily debated, the majority of published studies suggest a poorer outcome in patients with KRAS mutations (Siena et al, 2009). Interestingly, patients with Lynch syndrome-associated CRC, representing only $2-3 \%$ of all CRC patients, are also found to have a higher frequency of KRAS mutation, yet they generally show a favourable clinical outcome (Oliveira et al, 2007; Green et al, 2009). Downstream of $K R A S$ in ERK/MAPK signalling lies $B R A F$, a gene that in sporadic disease is mutated in $\sim 10 \%$ of $\mathrm{CRC}$ and is more highly associated with tumours showing microsatellite instability (MSI) (Bos et al, 1987; Jass, 2007). Few reports have investigated the prognostic effect of $B R A F$ in CRC; however, evidence points to a worse prognosis in patients with mutations in this gene (Samowitz et al, 2005; French et al, 2008; Ogino et al, 2009). 
Current regimens for patients with metastatic CRC include antiEGFR monoclonal antibodies such as cetuximab and panitumumab, both functioning to block the binding of ligands to EGFR, thereby downregulating ERK/MAPK and PI3K/PTEN/AKT pathway signalling (Amado et al, 2008; Giusti et al, 2008; Segal and Saltz, 2009). New evidence suggests that patients with KRAS, BRAF or PTEN mutations experience fewer clinical responses to these drugs, compared with patients with wild-type tumours; moreover, molecular analysis, particularly of KRAS, is routinely being performed in standard molecular pathology laboratories (Lievre et al, 2006; Amado et al, 2008; Di Nicolantonio et al, 2008; Karapetis et al, 2008; Au et al, 2009; Jimeno et al, 2009; Sartore-Bianchi et al, 2009). As $30-40 \%$ of patients with CRC exhibit mutations in one of these genes in a mutually exclusive manner, their potential for receiving such targeted agents is substantially decreased (Jass, 2007; Di Nicolantonio et al, 2008).

Taken together, the identification of novel prognostic and predictive factors, which consider the heterogeneous molecular background of CRC, particularly with regard to KRAS and BRAF gene status, is warranted. In 2000, a new member of the ERK/ MAPK pathway, T-cell-originated protein kinase (TOPK), also known as PDZ-binding kinase, was identified (Abe et al, 2000; Gaudet et al, 2000). T-cell-originated protein kinase was described as a MAPKK-like protein involved in p38MAPK and JNK signalling, possibly in a cell-type-dependent manner, and was more recently found to be involved in the ERK/MAPK pathway (Matsumoto et al, 2004; Nandi et al, 2004; Ayllon and O'Connor, 2007; Oh et al, 2007). T-cell-originated protein kinase is overexpressed in highly proliferating normal tissues, foetal tissues and in a wide variety of tumours in vitro, whereas the inhibition of TOPK is shown to lead to apoptosis in breast and melanoma cell lines (Simons-Evelyn et al, 2001; Zhao et al, 2001; Matsumoto et al, 2004; Nandi et al, 2004; Dougherty et al, 2005; Park et al, 2006; Zykova et al, 2006). Most recently, Herrero-Martin et al (2009) evaluated TOPK expression in Ewing sarcoma cell lines and found that the inhibition of TOPK led to a decrease in the proliferation rate and an important change in cell growth, indicating that TOPK could have a significant role in Ewing sarcoma biology. Zhu et al (2007) systematically assessed this novel molecule in CRC and confirmed its oncogenic potential in vitro and in vivo. Importantly, they found that, unlike other MEKs that undergo negative phosphorylation loops between themselves and ERK, TOPK could promote malignant transformation by exerting a positive feedback loop on ERK2 activity. However, the prognostic and predictive effect of TOPK in patients with CRC has to date not been explored.

Given its central involvement in ERK/MAPK signalling, we hypothesised that TOPK overexpression is significantly related to KRAS and $B R A F$ mutations, thereby implicating this gene in the poorer outcome of patients, both in terms of prognosis and response to anti-EGFR therapies. The aim of our study was, first, to determine using two randomised subgroups $(n=543$ and $n=501)$ whether TOPK expression leads to reproducible associations with clinicopathological features by immunohistochemistry (IHC) and, second, to determine according to KRAS and BRAF gene status the prognostic effect of TOPK on 222 sporadic and 71 Lynch syndrome-associated CRC patients, as well as the prognostic and predictive value of TOPK in 45 metastatic CRC patients treated with anti-EGFR agents, cetuximab and panitumumab.

\section{METHODS}

\section{Patients}

Sporadic CRC patients (Groups 1 and 2) A total of 1420 primary pre-operatively untreated, unselected sporadic CRC patients treated at the University Hospital of Basel between 1987 and 1996 were included in this study. Haematoxylin and eosin-stained slides were retrospectively collected from the Institute of Pathology, University Hospital of Basel, the Institute of Clinical Pathology, Basel, Switzerland and from the Institute of Pathology, Stadtspital Triemli, Zürich, Switzerland. Histopathological criteria were reviewed by an experienced gastrointestinal pathologist (LT) and included tumour diameter, $\mathrm{pT}$ and $\mathrm{pN}$ classification, grade of differentiation, histological subtype, presence of vessel invasion, tumour border configuration (pushing/expanding or infiltrating) and presence of peritumoural lymphocytic inflammation at the invasive tumour front (Jass et al, 1986). Clinical data including patient age at diagnosis, tumour location and follow-up, local recurrence, distant metastasis and post-operative therapy were retrieved from the patient records, where available. Censored observations included patients who were alive at the last follow-up, those who died for reasons other than CRC or were lost to followup. Median survival time was 76 (95\% CI 47 - 137) months; median follow-up was 60.3 months.

Lynch syndrome-associated CRC patients (Group 3) In all, 94 patients with genetically confirmed Lynch syndrome-associated CRC identified from the Swiss Cancer Registry were included in this study. Histopathological criteria were reviewed and included pT, pN, pM classifications and grade of differentiation. Clinical data including patient age at diagnosis, tumour location and follow-up were retrieved from patient records. Censored observations included patients who were alive at last follow-up, those who died for reasons other than CRC or were lost to follow-up. Followup period ranged from 0 to 74 years and median follow-up time was 7.1 years (95\% CI $5.4-8.7)$.

Metastatic CRC patients (Group 4) A total of 46 consecutive patients with histologically confirmed metastatic CRC treated at the Oncology Institute of Southern Switzerland, Bellinzona, Switzerland with cetuximab or panitumumab-based regimens were entered into this study. Cetuximab was administered at a standard loading dose of $400 \mathrm{mg} \mathrm{m}^{-2}$ over $2 \mathrm{~h}$, followed by weekly dose of $250 \mathrm{mg} \mathrm{m}^{-2}$ over $1 \mathrm{~h}$. Panitumumab $\left(6 \mathrm{mg} \mathrm{kg}^{-1}\right)$ was administered intravenously every 2 weeks until progression was allocated in two patients who were refractory to oxaliplatin-based and irinotecan-based regimens. With the exception of two patients who received cetuximab as frontline therapy, the others had failed at least one previous chemotherapy regimen. For those patients who progressed on irinotecanbased regimens, cetuximab was administered in combination with these regimens given at the same dose and schedule. Treatment was continued until progressive disease (PD) or toxicity occurred, according to standard criteria. Clinical response was assessed every 6-8 weeks with radiological examination (computerised tomodensitometry or magnetic resonance imaging).

Figure I Study design. (A) 1420 sporadic colorectal cancers (CRCs) mounted onto tissue microarrays (TMA) underwent immunohistochemistry (IHC) for TOPK and were then subdivided into Group I $(n=1198)$ and Group $2(n=245)$ on the basis of the availability of paraffin-embedded material. Excluding I 54 cases, Group I was randomised into two matched subgroups ( $n=543$ and 50 I), then used to define 'diffuse' and 'patchy' TOPK expression and to test associations of TOPK with cliniopathological features. In Group 2, 23 cases were excluded. A total of 222 cases with evaluable TOPK IHC were analysed for microsatellite instability (MSI), KRAS and BRAF. The prognostic value of TOPK stratified by KRAS and BRAF gene status was determined. (B) TOPK IHC staining was assessable in 7I of 94 Lynch syndrome-associated CRC patients in Group 3. T-cell-originated protein kinase expression was related to KRAS and BRAF mutation, clinicopathological features and cancer-specific survival time. (C) TOPK IHC was assessable in 45 of 46 metastatic CRC patients, whereas investigations of MSI, KRAS, BRAF and PTEN were performed. The prognostic and predictive value of TOPK in metastatic CRC patients treated with anti-EGFR agents was evaluated. 
Prognostic and predictive value of TOPK in colorectal cancer

I Zlobec et al

The Response Evaluation Criteria in Solid Tumours (RECIST) were adopted for evaluation, and objective tumour response was classified into complete response, partial response (PR), stable disease (SD) and PD. Follow-up time ranged from 0 to 8 years, with a median of 2.0 years and a median survival time of 2.4 $(95 \%$ CI $2.0-3.4)$ years.

A

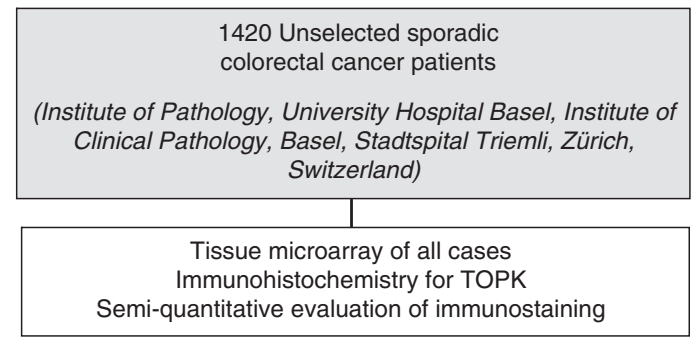
Corresponding paraffin-embedded materia
not available $(n=1198)$

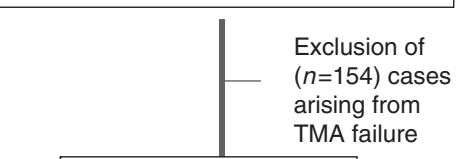

GROUP 1:

Randomisation of 1044

cases into two subgroups

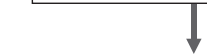

Subgroup A $(n=543)$

\section{Subgroup B $(n=501)$}

Aim Group 1:

Determine cutoff score for 'diffuse' and 'patchy' TOPK expression and test reproducibility of association of TOPK with clinicopathological features
(Swiss Cancer Registry)

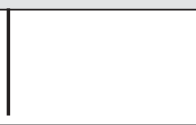

Tissue microarray with multiple tumor punches. Immunohistochemistry for TOPK

Average TOPK expression per case obtained. Cases classified as 'patchy' or 'diffuse' using the cutoff score determined from Group 1 subgroup A

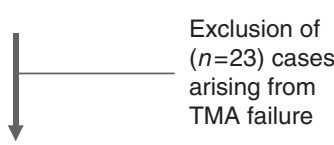

71 Cases evaluable for TOPK. 70 Paraffin-embedded tissue blocks available for molecular analysis of $K R A S$ and $B R A F$

Aim Group 3 :

Determine association of TOPK expression with KRAS, BRAF, clinicopathological features and survival time in hereditary

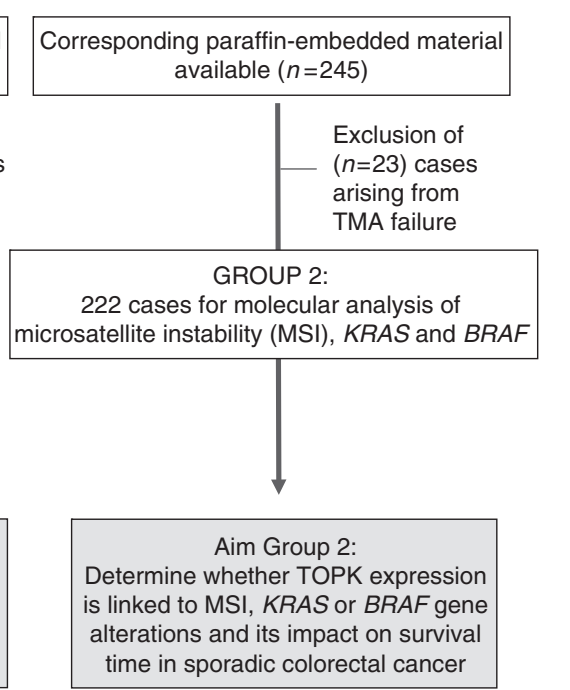

C

46 Consecutive patients with metastatic disease treated with cetuximab or panitumumab

(Institute of Southern Switzerland)
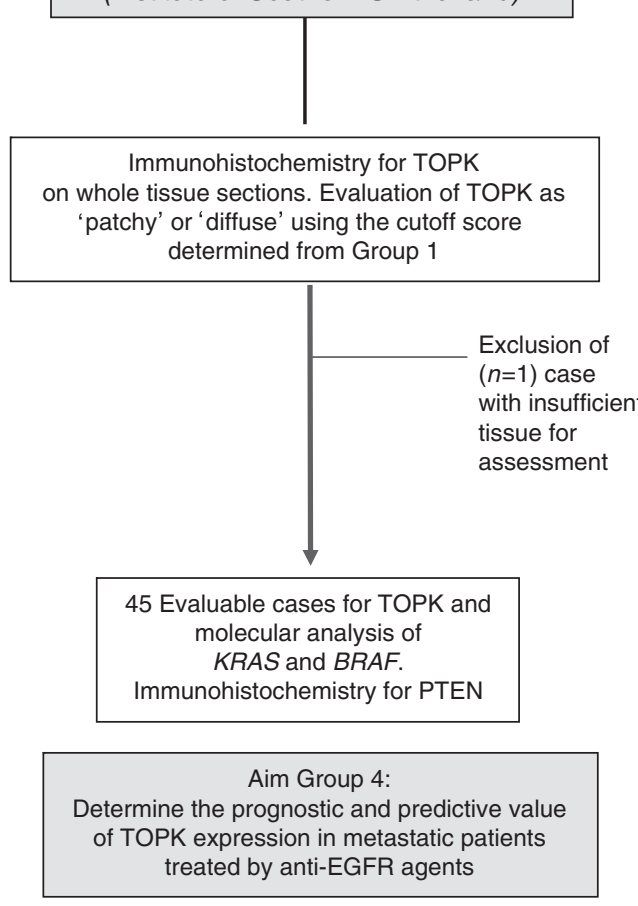
colorectal cance

colorectal cancer



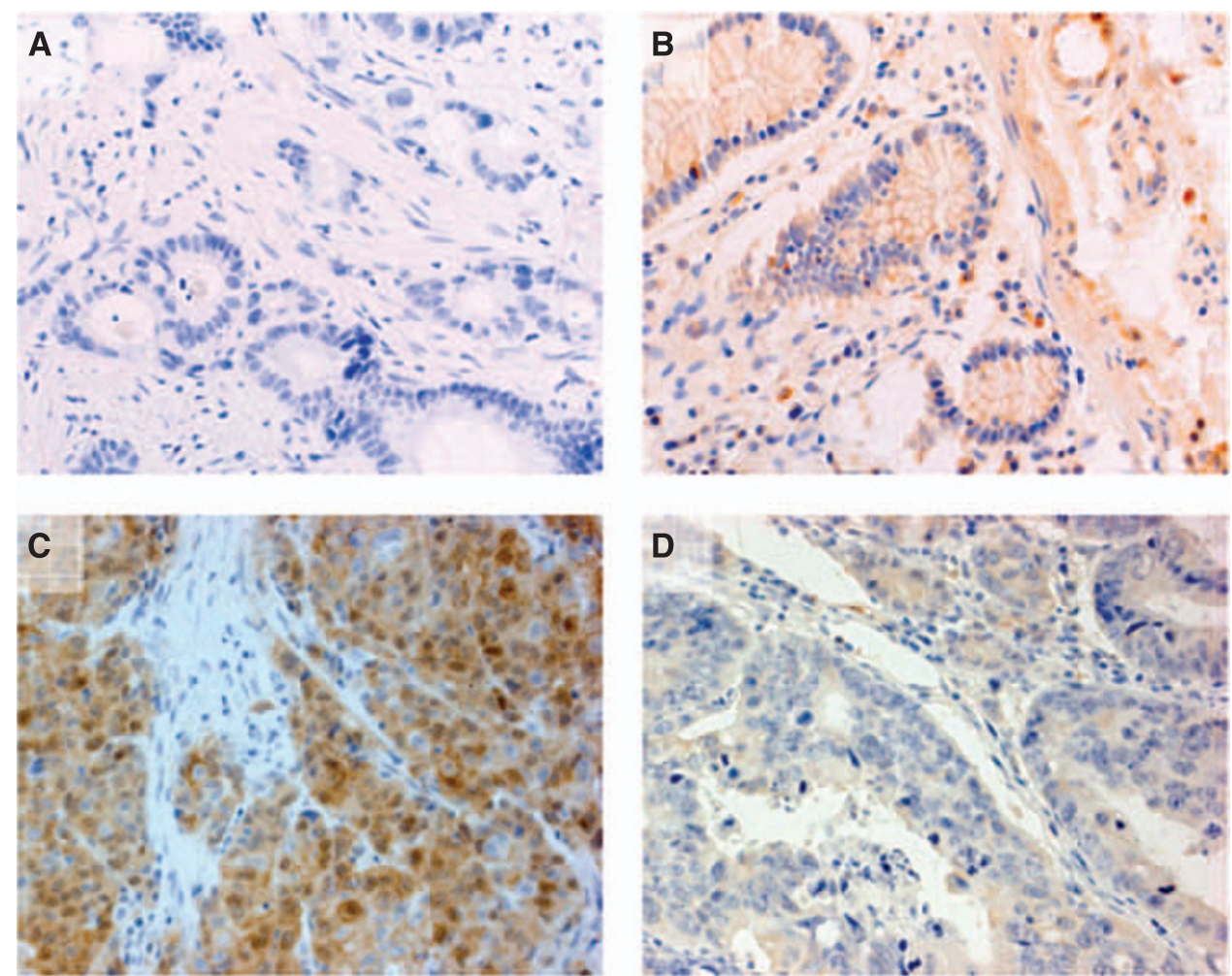

Figure 2 Representative photomicrographs $(\times 40)$ after immunohistochemistry staining with anti-TOPK antibody. (A) Colorectal cancer used as a negative control with the primary antibody omitted; (B) normal colonic mucosa with negligible cytoplasmic TOPK staining; (C) diffuse cytoplasmic TOPK staining in $>90 \%$ of colorectal tumour cells; and (D) patchy cytoplasmic staining of TOPK in $\leqslant 90 \%$ of colorectal tumour cells.

\section{Specimen characteristics}

For sporadic CRC patients (Groups 1 and 2), a previously described single-punch tissue microarray was constructed including all 1420 tumours and 57 normal mucosa samples as control (Sauter et al, 2003; Zlobec et al, 2008). Of the 1420 tumours, paraffin-embedded surgical resection specimens were available for 245 cases, which were retrospectively collected from the archives of the Institute of Pathology, University Hospital Basel, Switzerland for subsequent molecular analysis. Second, a multiple-punch tissue microarray including all 94 patients with Lynch syndrome-associated CRCs was constructed. Briefly, tissue blocks were retrieved from the Research Group Human Genetics, Department of Biomedicine, University of Basel. Haematoxylin and eosin slides were re-evaluated and representative areas from the tumour centre, tumour invasive front and adjacent normal mucosa (if available) were identified using a felt-tip pen. Tissue punches $0.6 \mathrm{~mm}$ in diameter were taken from these areas and brought into one recipient paraffin block $(3 \times 2.5 \mathrm{~cm})$ using a homemade semi-automated tissue arrayer. The final tissue microarray contained 297 tissues, taken from 101 different tissue blocks, and included 135 punches from the tumour centre, 78 from the tumour front and 84 samples of normal tissue. Third, for patients with metastatic disease, the corresponding paraffinembedded tissue blocks were retrospectively collected and whole-tissue sections were cut at $4 \mu \mathrm{m}$.

\section{Assay methods}

Immunohistochemistry Immunohistochemistry was carried out for all tumour specimens from Groups 1 to 4 and for normal mucosa samples using anti-TOPK antibody. Tissue microarrays and whole-tissue sections were dewaxed and rehydrated in $\mathrm{dH}_{2} \mathrm{O}$. After pressure cooker-mediated antigen retrieval in $0.001 \mathrm{M}$ EDTA
( $\mathrm{pH} 8.0$ ), endogenous peroxidase activity was blocked using $0.5 \%$ $\mathrm{H}_{2} \mathrm{O}_{2}$. Sections were incubated with $10 \%$ normal goat serum for $20 \mathrm{~min}$. After incubation with primary antibody (PBK/TOPK, rabbit polyclonal, dilution 1:50, Cell Signalling, Danvers, MA, USA), sections were incubated with HRP-conjugated secondary antibody (DakoCytomation, Glostrup, Denmark) for $30 \mathrm{~min}$ at room temperature, immersed in 3-amino-9-ethylcarbazole plus substrate-chromogen (DakoCytomation) for $30 \mathrm{~min}$ and counterstained with haematoxylin. Negative control tissues underwent the same protocol with the primary antibody omitted. Tumour cell immunoreactivity was evaluated by an experienced gastrointestinal pathologist (AL) blinded to clinical end points. Tumour cell staining for TOPK was predominantly observed in the cytoplasm, rather than in the nucleus or membrane. The percentage of positive tumour cells per case was scored. Staining intensity was not considered. The inter-observer variability of TOPK scores was assessed on one tissue microarray slide containing 456 cases by a second independent pathologist ( $\mathrm{MH}$ ) from an external institution and blinded to clinicopathological features.

Molecular analyses For groups 2, 3 and 4, MSI analysis along with KRAS (exon 2, codons 12 and 13) and BRAF (exon 15, codon 600) mutational investigations was performed as detailed previously (Frattini et al, 2007; Lugli et al, 2009). Microsatellite stable and MSI-low status were defined as instability at 0 and 1 markers, respectively. Microsatellite instability-high was characterised by the presence of instability in $\geqslant 2$ markers (Umar et al, 2004).

\section{Study design}

The study design is outlined in Figure 1. For study groups 1-3, excluded cases were those resulting from tissue microarray failure, that is, insufficient tissue for evaluation or $<50 \%$ tumour/punch. 
Table I Group I: immunohistochemical expression of TOPK (patchy or diffuse) and association with clinicopathological features in both randomized subgroups $A$ and $B$

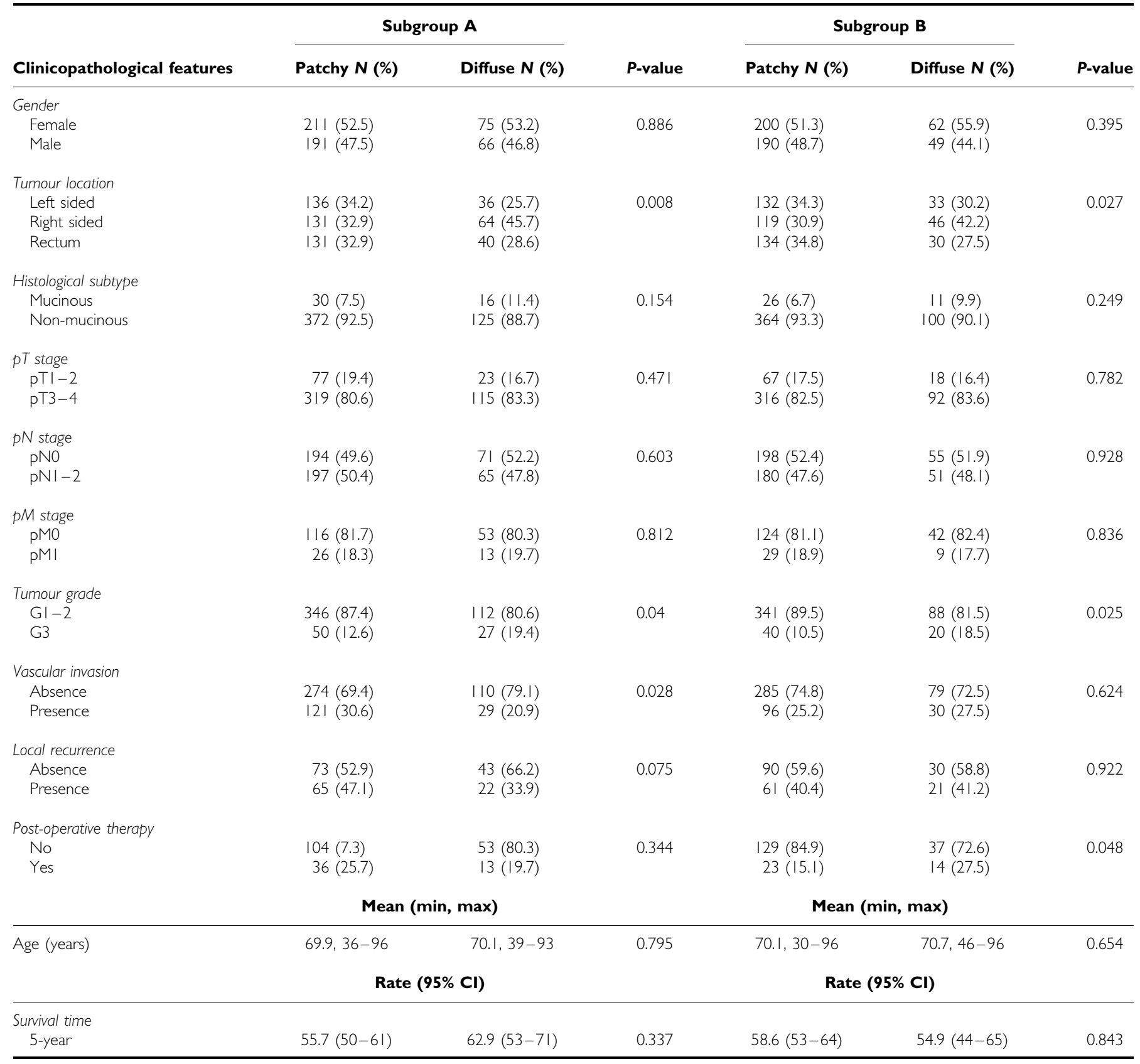

Abbreviations: $\mathrm{Cl}=$ confidence interval; $\mathrm{N}=$ frequency; TOPK $=$ T-cell-originated protein kinase.

The 1420 sporadic CRCs mounted onto the tissue microarray underwent IHC for TOPK and staining was evaluated semiquantitatively. These cases were subdivided into two groups on the basis of the availability of corresponding paraffin-embedded material for subsequent DNA extraction (Figure 1A). Group 1 included cases without available tumour blocks $(n=1198)$, whereas Group 2 represented cases with available archival paraffin-embedded material $(n=245)$.

After exclusion of 154 cases, Group 1 was further randomised into two matched subgroups containing 543 and 501 patients each. The appropriate IHC cutoff score for TOPK for all study groups was determined using subgroup A. Second, the reliability of TOPK expression and its association with clinicopathological features could be determined by analysing both subgroups independently.
After exclusion of 23 cases from Group 2, 222 cases underwent molecular investigations for MSI, KRAS and BRAF. The aim of this study group was to determine the prognostic value of TOPK in CRCs, with subgroup analysis by KRAS and BRAF mutation. Multivariable cancer-specific survival time models were evaluated by including candidate variables such as age, sex, $\mathrm{pT}$ and $\mathrm{pN}$ classification, vascular invasion and MSI status.

A total of 23 cases were excluded from Group 3 (Figure 1B). The remaining 71 Lynch syndrome-associated CRCs underwent molecular analysis for KRAS and BRAF. The association of TOPK expression with mutational status of KRAS and BRAF, clinicopathological features and cancer-specific survival time, was assessed.

One case of metastatic CRC was excluded from Group 4 because of insufficient material for adequate assessment of TOPK 
Table 2 Group 2: immunohistochemical expression of TOPK (patchy or diffuse) and association with clinicopathological and molecular features in sporadic colorectal cancer

\begin{tabular}{|c|c|c|c|}
\hline \multirow[b]{2}{*}{ Clinicopathological features } & \multicolumn{3}{|c|}{ Group $2(N(\%))$} \\
\hline & Patchy & Diffuse & $P$-value \\
\hline \multicolumn{4}{|l|}{ Gender } \\
\hline Female & $87(54.7)$ & $28(44.4)$ & 0.167 \\
\hline Male & $72(45.3)$ & $35(55.6)$ & \\
\hline \multicolumn{4}{|l|}{ Tumour location } \\
\hline Left sided & $42(26.4)$ & $18(28.6)$ & 0.05 \\
\hline Right sided & $47(29.6)$ & $27(42.9)$ & \\
\hline Rectum & $70(44.0)$ & $18(28.6)$ & \\
\hline \multicolumn{4}{|l|}{ Histological subtype } \\
\hline Mucinous & $6(3.8)$ & $8(12.7)$ & 0.027 \\
\hline Non-mucinous & $153(96.2)$ & $55(87.3)$ & \\
\hline \multicolumn{4}{|l|}{ pT stage } \\
\hline $\mathrm{pTI}-2$ & $36(22.8)$ & $13(21.3)$ & 0.815 \\
\hline PT3-4 & $122(77.2)$ & $48(78.7)$ & \\
\hline \multicolumn{4}{|l|}{ pN stage } \\
\hline pNo & $84(54.6)$ & $36(59.0)$ & 0.552 \\
\hline $\mathrm{pNI}-2$ & $70(45.5)$ & $25(41.0)$ & \\
\hline \multicolumn{4}{|l|}{ Tumour grade } \\
\hline GI-2 & I 54 (97.5) & $54(88.5)$ & 0.012 \\
\hline G3 & $4(2.5)$ & $7(11.5)$ & \\
\hline \multicolumn{4}{|l|}{ Vascular invasion } \\
\hline Absence & I $12(70.9)$ & $43(70.5)$ & 0.954 \\
\hline Presence & $46(29.1)$ & $18(29.5)$ & \\
\hline \multicolumn{4}{|l|}{ KRAS } \\
\hline Wild type & $117(76.5)$ & $36(63.2)$ & 0.054 \\
\hline Mutation & $36(23.5)$ & $21(36.8)$ & \\
\hline \multicolumn{4}{|l|}{ BRAF } \\
\hline Wild type & $129(89.6)$ & $39(72.2)$ & 0.002 \\
\hline Mutation & $15(10.4)$ & $15(27.8)$ & \\
\hline \multicolumn{4}{|l|}{ KRAS/BRAF } \\
\hline Both wild type & $108(67.9)$ & $27(42.9)$ & $<0.001$ \\
\hline KRAS or BRAF mutation & $51(32.1)$ & $36(57.1)$ & \\
\hline \multicolumn{4}{|l|}{ Microsatellite status } \\
\hline Stable/low & $126(79.3)$ & $48(76.2)$ & 0.618 \\
\hline \multirow[t]{2}{*}{ High } & $33(20.8)$ & $15(23.8)$ & \\
\hline & \multicolumn{2}{|c|}{ Mean (min, max) } & \\
\hline \multicolumn{4}{|l|}{ Age (years) } \\
\hline Mean, range & $67.6,43-95$ & $69.7,44-89$ & 0.156 \\
\hline & Rate & $5 \% \mathrm{Cl})$ & \\
\hline
\end{tabular}

5-year survival time

All patients

$54.6(47-63) \quad 52.3(39-64)$

Abbreviations: $\mathrm{Cl}=$ confidence interval; $\mathrm{N}=$ frequency; TOPK $=$ T-cell-originated protein kinase.

expression (Figure 1C). Immunohistochemistry for PTEN and molecular investigations of MSI, KRAS and BRAF were previously performed (Frattini et al, 2007). The prognostic and predictive value of TOPK in this group of patients was analysed, with specific end points of interest being cancer-specific survival time and objective tumour response to anti-EGFR agents.
The use of all patient material was approved by local Ethics Committees.

\section{Statistical analysis methods}

Associations of TOPK with categorical features were investigated by Chi-Square and Fisher's Exact tests where appropriate, and by Student's $t$-test for age. Survival analysis was performed using the Kaplan-Meier method, log-rank test and by multiple Cox regression analysis after verification of the proportional hazards assumption. The appropriate number of variables to be included in regression models was dependent on the frequency of patient deaths in each analysis. We included 1 variable per 10 deaths, to prevent overfitting. Differences in TOPK expression between normal colonic mucosa and tumour were determined using Wilcoxon's rank-sum test for medians. The most clinically relevant cutoff score for TOPK was determined on subgroup A by receiver operating characteristic (ROC) curve analysis for end point survival/death. To prevent overfitting, re-sampling of data was performed by bootstrapping 200 times. The inter-observer variability of TOPK staining was assessed using the intra-class correlation coefficient (ICC), with values of $\geqslant 0.8$ indicating excellent agreement. Missing clinicopathological data were assumed to be at random. No imputation was performed; rather, only patients with complete data for all features were included in multivariable analyses. $P$-values $<0.05$ were considered to be statistically significant.

\section{RESULTS}

\section{TOPK expression in normal colon versus sporadic CRC}

T-cell-originated protein kinase expression in 57 normal colonic mucosa samples was compared with sporadic CRCs from Group 1 $(n=1044)$. T-cell-originated protein kinase was highly overexpressed in tumours with a median of $90 \%$ positive cell staining compared with $5 \%$ positive cell staining in normal tissue $(P<0.001)$.

\section{Inter-observer agreement of TOPK scoring and determination of the cutoff score in CRC}

Re-evaluation of one tissue microarray slide ( $n=456$ CRCs) by a second independent pathologist from an external institution using the same semi-quantitative scoring method resulted in ICC $=0.92$, indicating excellent agreement. Having established that the evaluation of TOPK staining was reproducible between observers, next, the most appropriate cutoff score to describe tumours as overexpressed for TOPK was evaluated. Using ROC curve analysis, the protein expression value with the highest sensitivity and specificity for patient survival was obtained for subgroup A (Group 1) and was found to be $90 \%$ positive for cell staining. This value also coincided with the median expression value of TOPK in sporadic CRCs in Group 1, hence tumours with $>90 \%$ positive cell staining for TOPK were considered 'diffuse', whereas cases with $\leqslant 90 \%$ were defined as 'patchy' (Figure 2). This definition was subsequently applied to all tumours in this study.

\section{Group 1: TOPK in sporadic CRC and clinicopathological information}

In subgroups A and B, 141 and 111 patients had a diffuse TOPK expression ( 26 and $28 \%$ of cases, respectively). In both randomised subgroups, diffuse TOPK expression was associated with tumour location (more right sided; $P=0.008$ and $P=0.027$ ) and with high tumour grade $(P=0.04$ and $P=0.025)$ (Table 1). 
A
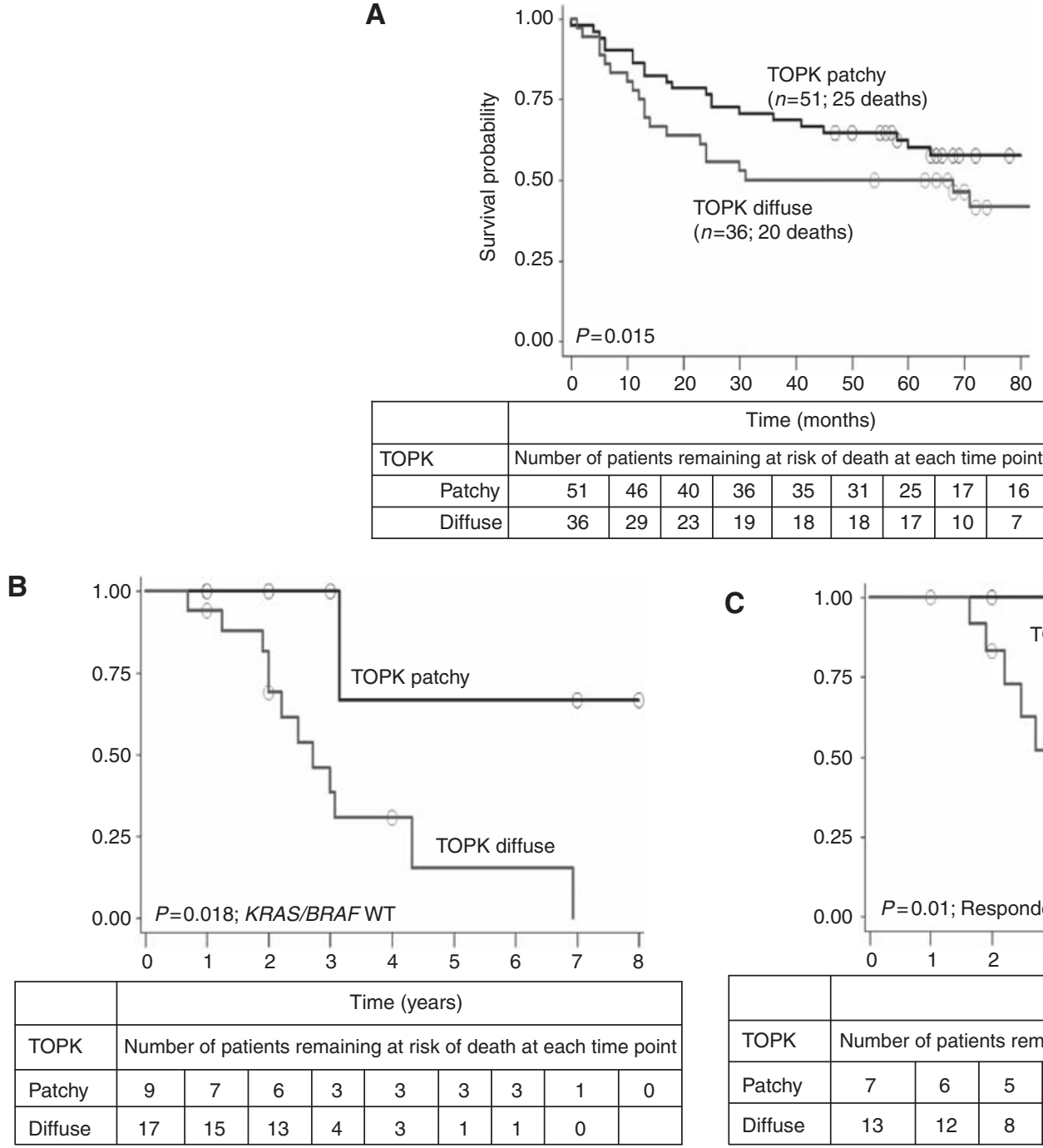

C

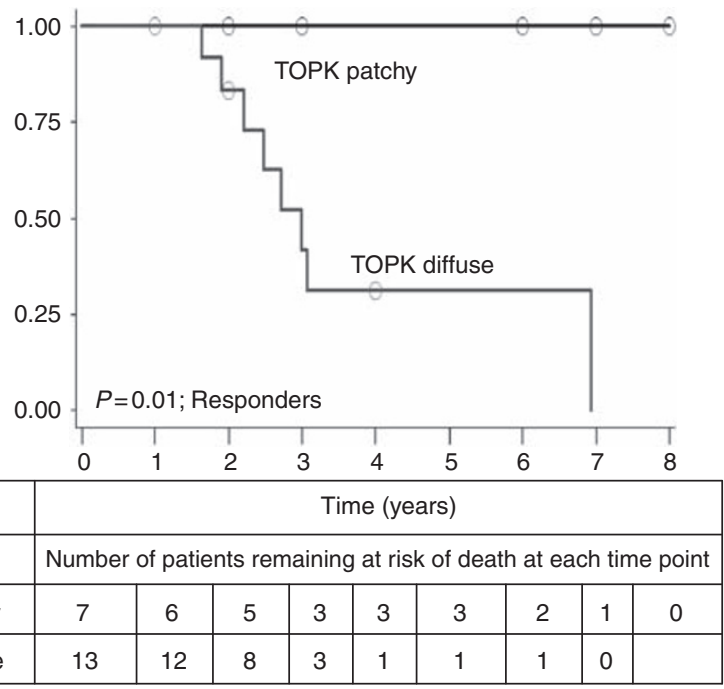

Figure 3 Kaplan-Meier survival curves (A) illustrating survival time differences among patients in Group 2 with KRAS or BRAF mutations stratified by TOPK expression, (B) of metastatic colorectal cancer patients illustrating the negative effect of diffuse TOPK expression on prognosis in patients with KRAS and BRAF wild-type tumours and $(\mathbf{C})$ of patients with stable disease or response to anti-EGFR therapy. Tables describe the number of patients at risk of death (alive) at each time point, beginning at the initial time of diagnosis when all patients are alive.

Table 3 Two multivariable analyses of TOPK expression in sporadic KRAS-mutated or BRAF-mutated colorectal cancer patients

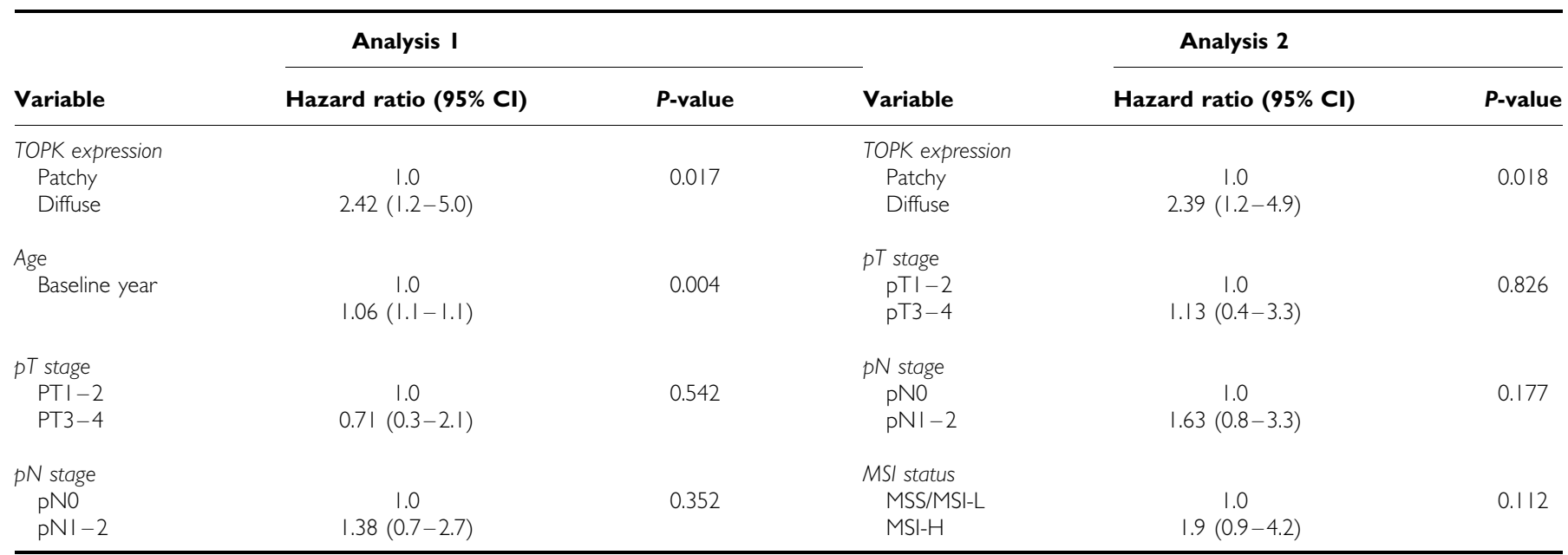

Abbreviations: $\mathrm{Cl}=$ confidence interval; $\mathrm{MSI}=$ microsatellite instability; $\mathrm{MSI}-\mathrm{L}=\mathrm{MSI}$ low; $\mathrm{MSI}-\mathrm{H}=\mathrm{MSI}$ high; $\mathrm{MSS}=$ microsatellite stable; TOPK = T-cell-originated protein kinase. 
Table 4 Group 3: immunohistochemical expression of TOPK (patchy or diffuse) and association with clinicopathological and molecular features in hereditary Lynch syndrome-associated colorectal cancers

\begin{tabular}{|c|c|c|c|}
\hline \multirow[b]{2}{*}{ Clinicopathological features } & \multicolumn{3}{|c|}{ Group 3 (N (\%)) } \\
\hline & Patchy & Diffuse & $P$-value \\
\hline \multicolumn{4}{|l|}{ Gender } \\
\hline Female & $22(53.7)$ & $18(60.0)$ & 0.635 \\
\hline Male & $19(46.3)$ & $12(40.0)$ & \\
\hline \multicolumn{4}{|l|}{ Tumour location } \\
\hline Left sided & $18(46.2)$ & | | (39.3) & 0.21 \\
\hline Right sided & $12(30.7)$ & $14(50.0)$ & \\
\hline Rectum & $9(23.1)$ & $3(10.7)$ & \\
\hline \multicolumn{4}{|l|}{ pT stage } \\
\hline PTI-2 & $12(31.6)$ & $2(6.9)$ & 0.014 \\
\hline рT3-4 & $26(68.4)$ & $27(93.1)$ & \\
\hline \multicolumn{4}{|l|}{ pN stage } \\
\hline $\mathrm{pNO}$ & $23(65.7)$ & $14(5 \mid .9)$ & 0.27 \\
\hline $\mathrm{pNI}-2$ & $12(34.3)$ & $13(48.2)$ & \\
\hline \multicolumn{4}{|l|}{ pM stage } \\
\hline $\mathrm{pMO}$ & $14(70.0)$ & $5(55.6)$ & 0.675 \\
\hline pMI & $6(30.0)$ & $4(44.4)$ & \\
\hline \multicolumn{4}{|l|}{ Tumour grade } \\
\hline $\mathrm{GI}-2$ & $24(72.7)$ & $18(64.3)$ & 0.478 \\
\hline G3 & $9(27.3)$ & $10(35.7)$ & \\
\hline \multicolumn{4}{|l|}{ KRAS } \\
\hline Wild type & $28(68.3)$ & $20(61.0)$ & 1.0 \\
\hline Mutation & $13(31.7)$ & $9(31.0)$ & \\
\hline \multicolumn{4}{|l|}{ BRAF } \\
\hline Wild type & $38(100.0)$ & $28(96.6)$ & 0.433 \\
\hline Mutation & $0(0.0)$ & I (3.5) & \\
\hline \multicolumn{4}{|l|}{ KRAS/BRAF } \\
\hline Both wild type & $28(68.3)$ & $19(65.5)$ & 1.0 \\
\hline KRAS or BRAF mutation & $13(31.7)$ & $10(34.5)$ & \\
\hline \multicolumn{4}{|l|}{ Microsatellite status } \\
\hline \multirow{3}{*}{$\begin{array}{l}\text { Stable/low } \\
\text { High }\end{array}$} & & & \\
\hline & 41 (57.3) & $30(42.3)$ & 0.192 \\
\hline & \multicolumn{2}{|c|}{ Mean (min, max) } & \\
\hline \multicolumn{4}{|l|}{ Age (years) } \\
\hline \multirow[t]{2}{*}{ Mean, range } & $45.3,24-73$ & $47.4,27-83$ & 0.492 \\
\hline & \multicolumn{2}{|c|}{ Rate $(95 \% \mathrm{Cl})$} & \\
\hline
\end{tabular}

5-year survival time

$\begin{array}{llll}\text { All patients } & 87.5(73-95) & 88.7(69-96) & 0.66\end{array}$

Abbreviations: $\mathrm{Cl}=$ confidence interval; $\mathrm{EGFR}=$ epidermal growth factor receptor $N=$ frequency; $T O P K=$ T-cell-originated protein kinase.

\section{Group 2: TOPK in sporadic CRC, molecular features and survival time}

In Group 2, TOPK was evaluable in 222 cases. Diffuse expression, observed in 63 patients (corresponding to $28 \%$ of cases), was linked to tumour location (more right-sided tumours; $P=0.05$ ), mucinuous histological subtype $(P=0.027)$ and poor tumour grade $(P=0.012)$ (Table 2$)$.

Mutational investigations gave analysable sequences in 198 cases for $B R A F$ and 210 cases for KRAS mutations. BRAF mutations were observed in 30 cases (15\%), whereas KRAS mutations occurred in
Table 5 Group 4: Immunohistochemical expression of TOPK (patchy or diffuse) and clinicopathological and molecular features in metastatic colorectal cancer patients treated with anti-EGFR therapy

\begin{tabular}{|c|c|c|c|}
\hline \multirow[b]{2}{*}{ Clinicopathological features } & \multicolumn{3}{|c|}{$N(\%)$} \\
\hline & Patchy & Diffuse & $P$-value \\
\hline \multicolumn{4}{|l|}{ Age (years) } \\
\hline Mean, range & $65.7,48-82$ & $60.7,26-79$ & 0.113 \\
\hline \multicolumn{4}{|l|}{ Gender } \\
\hline Female & $8(36.4)$ & $9(39.1)$ & 0.848 \\
\hline Male & $14(63.6)$ & $14(60.9)$ & \\
\hline \multicolumn{4}{|l|}{ Clinical response } \\
\hline Progressive disease & $13(59.1)$ & $10(43.5)$ & 0.528 \\
\hline Partial response & $4(18.2)$ & $7(30.4)$ & \\
\hline Stable disease & $5(22.7)$ & $6(26.1)$ & \\
\hline \multicolumn{4}{|l|}{ KRAS codon 12 and 13} \\
\hline Wild type & $13(59.1)$ & $19(82.6)$ & 0.082 \\
\hline Mutation & $9(40.9)$ & $4(17.4)$ & \\
\hline \multicolumn{4}{|l|}{ BRAF codon 600} \\
\hline Wild type & $20(90.9)$ & $21(91.3)$ & 1.0 \\
\hline Mutation & $2(9.1)$ & $2(8.7)$ & \\
\hline \multicolumn{4}{|l|}{ KRAS/BRAF } \\
\hline Both wild type & | | (50.0) & $17(73.9)$ & 0.098 \\
\hline KRAS or BRAF mutation & II (50.0) & $6(26.1)$ & \\
\hline \multicolumn{4}{|l|}{ Microsatellite status } \\
\hline Stable/low & $22(100.0)$ & $22(100.0)$ & \\
\hline High & & & \\
\hline \multicolumn{4}{|l|}{ EGFR amplification } \\
\hline No copy number gain & $4(19.1)$ & $3(13.0)$ & 0.693 \\
\hline Copy number gain & $17(81.0)$ & $20(87.0)$ & \\
\hline \multicolumn{4}{|l|}{ PIJKCA } \\
\hline Loss & $19(86.4)$ & $20(87.0)$ & 1.0 \\
\hline Overexpression & $3(13.6)$ & $3(13.0)$ & \\
\hline \multicolumn{4}{|l|}{ PTEN } \\
\hline Loss & | | (50.0) & $6(26.1)$ & 0.09 \\
\hline \multirow[t]{2}{*}{ Overexpression } & II (50.0) & $17(73.9)$ & \\
\hline & \multicolumn{2}{|c|}{ Rate $(95 \% \mathrm{Cl})$} & \\
\hline \multicolumn{4}{|l|}{ 5-year survival time } \\
\hline All patients & $34.5(11-60)$ & $13.5(\mid-40)$ & 0.473 \\
\hline Either KRAS or BRAF mutation & $18.2(3-44)$ & $\mid 6.7(0-5 \mid)$ & 0.887 \\
\hline Both wild type KRAS and BRAF & $66.7(5-95)$ & $15.3(1-45)$ & 0.018 \\
\hline Stable disease or response & 100 & $31.3(8-59)$ & 0.01 \\
\hline
\end{tabular}

Abbreviations: $\mathrm{Cl}=$ confidence interval; $\mathrm{EGFR}=$ epidermal growth factor receptor: $N=$ frequency; $\mathrm{TOPK}=\mathrm{T}$-cell-originated protein kinase.

57 cases $(27 \%)$. Mutations in BRAF $(P=0.002)$ and KRAS $(P=0.054)$ occurred more frequently in patients with diffuse TOPK staining compared with patients with wild-type tumours. As KRAS and BRAF mutations were mutually exclusive, the relationship of TOPK with either KRAS or BRAF mutation was evaluated. The diffuse expression found in 36 of $63(57.1 \%)$ patients was significantly associated with mutation in either $K R A S$ or $B R A F$, compared with $32.1 \%$ of patients with a patchy expression $(P<0.001)$.

Among patients with KRAS or BRAF mutations, those with diffuse TOPK expression had a significantly worse prognosis compared with patients with a patchy expression $(P=0.015)$ (Figure 3A). The relative risk of death for patients with KRAS or BRAF mutations was 2.22 (95\% CI $1.1-4.4)$ compared with those showing no mutation in either gene. In multivariate survival 
analysis with age, $\mathrm{pT}$ classification and $\mathrm{pN}$ classification, TOPK expression maintained a significant adverse effect on outcome $(P=0.017 ; \mathrm{HR}=2.42(95 \% \mathrm{CI} 1.2-5.0))$, as well as after adjusting for the prognostic effects of $\mathrm{pT}$ classification, $\mathrm{pN}$ classification and MSI status $(P=0.018 ; \mathrm{HR}=2.39(95 \%$ CI $1.2-4.9))$ (Table 3$)$.

\section{Group 3: TOPK in hereditary Lynch syndrome-associated CRC}

T-cell-originated protein kinase expression could be assessed in 71 patients with Lynch syndrome-associated CRC. Of the 30 patients with a diffuse TOPK expression (41\% of cases), 27 (93.1\%) had pT3 or pT4 tumours compared with $68.2 \%$ of patients with a patchy expression $(P=0.014)$. KRAS mutations were found in 22 (31\%) patients, whereas mutation in BRAF was noted in only one case of genetically confirmed Lynch syndrome. No association of TOPK was observed with either prognosis or KRAS mutation status (Table 4).

\section{Group 4: TOPK in metastatic CRC patients treated with anti-EGFR therapy}

Of the 45 metastatic patients treated with cetuximab or panitumumab with evaluable TOPK staining, a wild-type KRAS and BRAF gene status was detected in $32(71.1 \%)$ and $41(91 \%)$ cases, respectively. Diffuse TOPK expression was observed in 19 (82.6\%) KRAS wild-type and 21 (91.3\%) BRAF wild-type tumours. A highly unfavourable outcome in patients with KRAS and BRAF wild-type tumours with overexpression of TOPK was noted $(P=0.018)$ (Figure 3B). No difference in TOPK staining was found between PTEN loss and overexpression, and the prognostic effect of diffuse TOPK staining in KRAS and BRAF wild-type patients was maintained after adjusting for PTEN status $(P=0.041)$. In total, 23 patients $(51.1 \%)$ had PD, $11(24.4 \%)$ had PR and 11 $(24.4 \%)$ had SD, with diffuse expression of TOPK occurring in 10 (43.5\%), $7(30.4 \%)$ and $6(26.1 \%)$ patients, respectively. Patients having SD or PR to anti-EGFR therapy but with diffuse TOPK expression suffered from poor outcome; in contrast, those with no overexpression of TOPK were alive or censored at 5-year follow-up $(P=0.01)$ (Figure 3C). T-cell-originated protein kinase expression was not of predictive value for response to anti-EGFR therapy, either in the entire cohort of patients or when stratified by KRAS and $B R A F$ mutation status (Table 5).

\section{DISCUSSION}

We report the association of diffuse TOPK expression with specific sporadic CRC features, namely, with right-sided tumour location and higher tumour grade in two large multicentric cohorts of patients and excellent inter-observer reproducibility of TOPK scores. Second, our findings point to the diffuse expression of TOPK as an adverse prognostic factor in patients with sporadic CRC with a KRAS or BRAF mutation and in metastatic patients with SD or PR after treatment with anti-EGFRbased regimens.

In sporadic CRC, diffuse TOPK expression was associated with the presence of KRAS or BRAF mutation, underlining the involvement of TOPK in ERK/MAPK signalling. In patients with either KRAS or BRAF mutations, diffuse expression of TOPK had an adverse effect on 5-year survival. In addition, this unfavourable effect of TOPK expression on outcome was maintained in multivariate analysis, suggesting that TOPK could represent an important prognostic factor in patients with KRAS-mutated or $B R A F$-mutated tumours (Andreyev et al, 1998; Samowitz et al, 2005; French et al, 2008; Ogino et al, 2009). Although KRAS mutations are frequently found in patients with Lynch syndromeassociated CRC despite their favourable prognosis, in this study, no association between TOPK expression and KRAS mutation was observed (Oliveira et al, 2007). The propensity for more right-sided, poorly differentiated cancers and poorer outcome in patients with KRAS or BRAF mutation was not found here, despite an association with a more advanced $\mathrm{pT}$ stage with diffuse TOPK staining. These results seem to indicate that involvement of TOPK in CRC may be limited to tumours of sporadic origin.

We report that in 45 metastatic CRC patients treated with antiEGFR agents and with wild-type KRAS and BRAF gene status, those expressing diffuse TOPK staining suffer from a significant adverse prognosis. In addition, TOPK expression seemed to be unmodified by PTEN status and maintained its adverse effect on outcome in KRAS or BRAF wild-type patients independently of the expression of this molecule. Furthermore, among patients with SD or those with objective response, a diffuse expression of TOPK may act as a highly unfavourable prognostic factor. Together, these results indicate that the activation of MAPK signalling is still possible at the level of TOPK, even in the context of wild-type KRAS and BRAF, and is unlikely because of loss of PTEN. Therefore, TOPK may act as a prognostic, rather than as a predictive, factor, suggesting that it may be important to consider its expression in metastatic CRC patients with a proficient molecular profile for positive response to anti-EGFR drugs.

Our results suggest that inhibition of TOPK could be beneficial for at least two groups of CRC patients together representing 30 $40 \%$ of all cases, namely, those with a KRAS or BRAF mutation and those with metastatic disease supported by several factors. T-celloriginated protein kinase is barely detectable in most normal adult tissues including normal colonic mucosa, whereas it is highly overexpressed in CRC (Zhu et al, 2007). Its detection by IHC leads to reproducible associations with clinicopathological features and its evaluation leads to excellent inter-observer agreement. As a MAPKK-like protein, it is a downstream molecule of KRAS and $B R A F$, both of which are associated with diffuse expression of TOPK (Roberts and Der, 2007). T-cell-originated protein kinase may itself be an effector of BRAF, as phosphorylation of TOPK by RAF has previously been shown (Yuryev and Wennogle, 2003). Therefore, inhibition of TOPK at this level of signalling may have a more significant impact on downregulating deregulated ERK/ MAPK signalling. Current MEK inhibitors have led to moderate results (Roberts and Der, 2007). Although blocking MEK1 should lead to a decrease in the phosphorylation of ERK1/2, this process is hindered by a negative feedback loop of ERK1/2 onto MEK1, making inhibition of this molecule to some extent counter productive (Ramos, 2008). T-cell-originated protein kinase, in contrast, has been described as an oncogenic MEK involved in a positive phosphorylation loop with ERK2 (Zhu et al, 2007). Therefore, inhibition of TOPK should be expected to successfully decrease the activation of ERK2 and thus its downstream transcription factors. Moreover, TOPK expression in this study seems to be independent of PTEN status. Considering recent evidence suggesting that PTEN mutation results in resistance to EGFR-targeted therapies (Sartore-Bianchi et al, 2009), the inhibition of TOPK in KRAS and BRAF wild-type patients could represent an approach to improve clinical outcome in patients with either PTEN wild-type or mutated cancers.

A limitation of this study is that information on cancer treatment was limited. Subgroup analysis produced results using relatively small sample sizes; therefore, these findings necessitate validation on larger patient cohorts. Nonetheless, our study gives valuable results for several reasons. Four groups of patients were included, representing sporadic, hereditary and metastatic CRC. Patients were treated in different centres and considerable corresponding clinicopathological data and follow-up could be obtained. Whole-tissue sections and two tissue microarrays were evaluated, the largest containing more than 1000 tumours, the second with multiple tissue punches taken from the same patient representing different tumour areas. Finally, the cohort of 
metastatic CRC was well characterised with respect to both clinicopathological treatment and molecular features.

Although several study groups have investigated the functional role of TOPK in different tumour types, this seems to be the first assessment of the prognostic and predictive value of this protein in CRC. In conclusion, TOPK seems to be a valuable prognostic factor in patients with sporadic CRC with KRAS or BRAF gene mutations, as well as in patients with metastatic disease who respond to antiEGFR therapies. If confirmed prospectively, the inhibition of TOPK may represent a novel avenue of investigation for targeted treatment in patients with CRC, especially for the early identifica-

\section{REFERENCES}

Abe Y, Matsumoto S, Kito K, Ueda N (2000) Cloning and expression of a novel MAPKK-like protein kinase, lymphokine-activated killer T-celloriginated protein kinase, specifically expressed in the testis and activated lymphoid cells. J Biol Chem 275: 21525-21531

Amado RG, Wolf M, Peeters M, Van Cutsem E, Siena S, Freeman DJ, Juan T, Sikorski R, Suggs S, Radinsky R, Patterson SD, Chang DD (2008) Wild-type KRAS is required for panitumumab efficacy in patients with metastatic colorectal cancer. J Clin Oncol 26: 1626-1634

Andreyev HJ, Norman AR, Cunningham D, Oates JR, Clarke PA (1998) Kirsten ras mutations in patients with colorectal cancer: the multicenter 'RASCAL' study. J Natl Cancer Inst 90: 675-684

Au HJ, Karapetis CS, O'Callaghan CJ, Tu D, Moore MJ, Zalcberg JR Kennecke H, Shapiro JD, Koski S, Pavlakis N, Charpentier D, Wyld D, Jefford M, Knight GJ, Magoski NM, Brundage MD, Jonker DJ (2009) Health-related quality of life in patients with advanced colorectal cancer treated with cetuximab: overall and $K R A S$-specific results of the NCIC CTG and AGITG CO.17 trial. J Clin Oncol 27: 1822-1828

Ayllon V, O'Connor R (2007) PBK/TOPK promotes tumour cell proliferation through p38 MAPK activity and regulation of the DNA damage response. Oncogene 26: $3451-3461$

Bos JL, Fearon ER, Hamilton SR, Verlaan-de Vries M, van Boom JH, van der Eb AJ, Vogelstein B (1987) Prevalence of ras gene mutations in human colorectal cancers. Nature 327: 293-297

Di Nicolantonio F, Martini M, Molinari F, Sartore-Bianchi A, Arena S, Saletti P, De Dosso S, Mazzucchelli L, Frattini M, Siena S, Bardelli A (2008) Wild-type $B R A F$ is required for response to panitumumab or cetuximab in metastatic colorectal cancer. J Clin Oncol 26: 5705-5712

Dougherty JD, Garcia AD, Nakano I, Livingstone M, Norris B, Polakiewicz R, Wexler EM, Sofroniew MV, Kornblum HI, Geschwind DH (2005) PBK/ TOPK, a proliferating neural progenitor-specific mitogen-activated protein kinase kinase. J Neurosci 25: $10773-10785$

Fang JY, Richardson BC (2005) The MAPK signalling pathways and colorectal cancer. Lancet Oncol 6: 322-327

Frattini M, Saletti P, Romagnani E, Martin V, Molinari F, Ghisletta M, Camponovo A, Etienne LL, Cavalli F, Mazzucchelli L (2007) PTEN loss of expression predicts cetuximab efficacy in metastatic colorectal cancer patients. Br J Cancer 97: 1139-1145

French AJ, Sargent DJ, Burgart LJ, Foster NR, Kabat BF, Goldberg R, Shepherd L, Windschitl HE, Thibodeau SN (2008) Prognostic significance of defective mismatch repair and BRAF V600E in patients with colon cancer. Clin Cancer Res 14: 3408-3415

Gaudet S, Branton D, Lue RA (2000) Characterization of PDZ-binding kinase, a mitotic kinase. Proc Natl Acad Sci USA 97: 5167-5172

Giusti RM, Shastri K, Pilaro AM, Fuchs C, Cordoba-Rodriguez R, Koti K, Rothmann M, Men AY, Zhao H, Hughes M, Keegan P, Weiss KD, Pazdur R (2008) US Food and Drug Administration approval: panitumumab for epidermal growth factor receptor-expressing metastatic colorectal carcinoma with progression following fluoropyrimidine-, oxaliplatin-, and irinotecan-containing chemotherapy regimens. Clin Cancer Res 14: $1296-1302$

Green RC, Parfrey PS, Woods MO, Younghusband HB (2009) Prediction of Lynch syndrome in consecutive patients with colorectal cancer. J Natl Cancer Inst 101: $331-340$

Herrero-Martin D, Osuna D, Ordonez JL, Sevillano V, Martins AS, Mackintosh C, Campos M, Madoz-Gurpide J, Otero-Motta AP, Caballero G, Amaral AT, Wai DH, Braun Y, Eisenacher M, Schaefer KL, Poremba C de Alava E (2009) Stable interference of EWS-FLI1 in an Ewing sarcoma tion of patients with a worse prognosis, although experiencing disease control after anti-EGFR drug administration.

\section{ACKNOWLEDGEMENTS}

This study was funded by the Krebsliga Beider Basel (LT, KH, AL), Oncosuisse (MF, KH), the Fondazione Ticinese per la Ricerca sul Cancro (MF) and the Krebsliga Zentralschweiz (MK, KH). The sponsors were not involved in study design, collection, analysis and interpretation of data. cell line impairs IGF-1/IGF-1R signalling and reveals TOPK as a new target. Br J Cancer 101: 80-90

Jass JR (2007) Classification of colorectal cancer based on correlation of clinical, morphological and molecular features. Histopathology 50: 113-130 Jass JR, Atkin WS, Cuzick J, Bussey HJ, Morson BC, Northover JM, Todd IP (1986) The grading of rectal cancer: historical perspectives and a multivariate analysis of 447 cases. Histopathology 10: 437-459

Jimeno A, Messersmith WA, Hirsch FR, Franklin WA, Eckhardt SG (2009) KRAS mutations and sensitivity to epidermal growth factor receptor inhibitors in colorectal cancer: practical application of patient selection. J Clin Oncol 27: $1130-1136$

Karapetis CS, Khambata-Ford S, Jonker DJ, O'Callaghan CJ, Tu D, Tebbutt NC, Simes RJ, Chalchal H, Shapiro JD, Robitaille S, Price TJ, Shepherd L, Au HJ, Langer C, Moore MJ, Zalcberg JR (2008) K-ras mutations and benefit from cetuximab in advanced colorectal cancer. $N$ Engl J Med 359: $1757-1765$

Lievre A, Bachet JB, Le Corre D, Boige V, Landi B, Emile JF, Cote JF, Tomasic G, Penna C, Ducreux M, Rougier P, Penault-Llorca F, LaurentPuig P (2006) KRAS mutation status is predictive of response to cetuximab therapy in colorectal cancer. Cancer Res 66: $3992-3995$

Lugli A, Karamitopoulou E, Panayiotides I, Karakitsos P, Rallis G, Peros G, Iezzi G, Spagnoli G, Bihl M, Terracciano L, Zlobec I (2009) CD8+ lymphocytes/ tumour-budding index: an independent prognostic factor representing a 'pro-/anti-tumour' approach to tumour host interaction in colorectal cancer. Br J Cancer 101(8): 1382-1392

Matsumoto S, Abe Y, Fujibuchi T, Takeuchi T, Kito K, Ueda N, Shigemoto K, Gyo K (2004) Characterization of a MAPKK-like protein kinase TOPK. Biochem Biophys Res Commun 325: $997-1004$

Nandi A, Tidwell M, Karp J, Rapoport AP (2004) Protein expression of PDZ-binding kinase is up-regulated in hematologic malignancies and strongly down-regulated during terminal differentiation of HL-60 leukemic cells. Blood Cells Mol Dis 32: 240-245

Ogino S, Nosho K, Kirkner GJ, Kawasaki T, Meyerhardt JA, Loda M, Giovannucci EL, Fuchs CS (2009) CpG island methylator phenotype, microsatellite instability, BRAF mutation and clinical outcome in colon cancer. Gut 58: $90-96$

Oh SM, Zhu F, Cho YY, Lee KW, Kang BS, Kim HG, Zykova T, Bode AM, Dong Z (2007) T-lymphokine-activated killer cell-originated protein kinase functions as a positive regulator of c-Jun-NH2-kinase 1 signaling and H-Ras-induced cell transformation. Cancer Res 67: $5186-5194$

Oliveira C, Velho S, Moutinho C, Ferreira A, Preto A, Domingo E, Capelinha AF, Duval A, Hamelin R, Machado JC, Schwartz Jr S, Carneiro F, Seruca R (2007) KRAS and BRAF oncogenic mutations in MSS colorectal carcinoma progression. Oncogene 26: 158-163

Park JH, Lin ML, Nishidate T, Nakamura Y, Katagiri T (2006) PDZ-binding kinase/T-LAK cell-originated protein kinase, a putative cancer/testis antigen with an oncogenic activity in breast cancer. Cancer Res 66: 9186- 9195

Ramos JW (2008) The regulation of extracellular signal-regulated kinase (ERK) in mammalian cells. Int J Biochem Cell Biol 40: 2707-2719

Roberts PJ, Der CJ (2007) Targeting the Raf-MEK-ERK mitogen-activated protein kinase cascade for the treatment of cancer. Oncogene 26: $3291-3310$

Samowitz WS, Sweeney C, Herrick J, Albertsen H, Levin TR, Murtaugh MA, Wolff RK, Slattery ML (2005) Poor survival associated with the BRAF V600E mutation in microsatellite-stable colon cancers. Cancer Res 65: 6063-6069 
Sartore-Bianchi A, Martini M, Molinari F, Veronese S, Nichelatti M, Artale S, Di Nicolantonio F, Saletti P, De Dosso S, Mazzucchelli L, Frattini M, Siena S, Bardelli A (2009) PIK3CA mutations in colorectal cancer are associated with clinical resistance to EGFR-targeted monoclonal antibodies. Cancer Res 69: 1851-1857

Sauter G, Simon R, Hillan K (2003) Tissue microarrays in drug discovery. Nat Rev Drug Discov 2: $962-972$

Segal NH, Saltz LB (2009) Evolving treatment of advanced colon cancer. Annu Rev Med 60: 207-219

Siena S, Sartore-Bianchi A, Di Nicolantonio F, Balfour J, Bardelli A (2009) Biomarkers predicting clinical outcome of epidermal growth factor receptor-targeted therapy in metastatic colorectal cancer. J Natl Cancer Inst 101(19): $1308-1324$

Simons-Evelyn M, Bailey-Dell K, Toretsky JA, Ross DD, Fenton R, Kalvakolanu D, Rapoport AP (2001) PBK/TOPK is a novel mitotic kinase which is upregulated in Burkitt's lymphoma and other highly proliferative malignant cells. Blood Cells Mol Dis 27: 825-829

Umar A, Boland CR, Terdiman JP, Syngal S, de la Chapelle A, Ruschoff J, Fishel R, Lindor NM, Burgart LJ, Hamelin R, Hamilton SR, Hiatt RA, Jass J, Lindblom A, Lynch HT, Peltomaki P, Ramsey SD, Rodriguez-Bigas MA, Vasen HF, Hawk ET, Barrett JC, Freedman AN, Srivastava S (2004)
Revised Bethesda Guidelines for hereditary nonpolyposis colorectal cancer (Lynch syndrome) and microsatellite instability. J Natl Cancer Inst 96: $261-268$

Yuryev A, Wennogle LP (2003) Novel raf kinase protein-protein interactions found by an exhaustive yeast two-hybrid analysis. Genomics 81: $112-125$

Zhao S, Dai J, Zhao W, Xia F, Zhou Z, Wang W, Gu S, Ying K, Xie Y, Mao Y (2001) PDZ-binding kinase participates in spermatogenesis. Int $J$ Biochem Cell Biol 33: 631-636

Zhu F, Zykova TA, Kang BS, Wang Z, Ebeling MC, Abe Y, Ma WY, Bode AM, Dong Z (2007) Bidirectional signals transduced by TOPK-ERK interaction increase tumorigenesis of HCT116 colorectal cancer cells. Gastroenterology 133: 219-231

Zlobec I, Terracciano L, Tornillo L, Gunthert U, Vuong T, Jass JR, Lugli A (2008) Role of RHAMM within the hierarchy of well-established prognostic factors in colorectal cancer. Gut 57: 1413-1419

Zykova TA, Zhu F, Lu C, Higgins L, Tatsumi Y, Abe Y, Bode AM, Dong Z (2006) Lymphokine-activated killer T-cell-originated protein kinase phosphorylation of histone $\mathrm{H} 2 \mathrm{AX}$ prevents arsenite-induced apoptosis in RPMI7951 melanoma cells. Clin Cancer Res 12: $6884-6893$ 